\title{
Using wavelet spectrum analysis to resolve breaking events in the wind wave time series
}

\author{
P. C. Liu ${ }^{1}$ and A. V. Babanin ${ }^{2}$ \\ ${ }^{1}$ NOAA Great Lakes Environmental Research Laboratory, Ann Arbor, Michigan 48105, USA \\ ${ }^{2}$ Swinburne University of Technology, School of Engineering and Science, Hawthorn, Victoria 3122, Australia
}

Received: 5 April 2004 - Revised: 1 June 2004 - Accepted: 11 June 2004 - Published: 3 November 2004

\begin{abstract}
This paper presents the development of a new approach, based on wavelet spectrum analysis, for the detection of breaking waves in a time series of surface wave fluctuations. The approach is shown to be capable of producing equivalent wave breaking statistics as field measurements based on detection of whitecaps at a fixed point of observation. This wavelet-based approach is applicable to both deep water and finite depth environments. Based on applications of this approach to the analysis of available field data, a novel classification of wave breaking processes that consists of incipient, developing, and subsiding phases is proposed.
\end{abstract}

Key words. Meteorology and atmospheric dynamics (waves and tides) - Oceanography; physical (instruments and techniques)

\section{Introduction}

The surfaces of oceans and lakes are rarely at rest. Wind waves are ubiquitous features on these surfaces, and so are breaking waves. As long as there are wind waves of all scales and all frequencies, there are breaking waves in the form of whitecaps woven in the midst of the wind waves. The study of wind waves relies on the measurements of surface fluctuations from a variety of instruments, such as wave staffs, accelerometers, pressure, acoustic, and laser sensors, among others. There are vast amounts of wave records accumulated over the past decades. Being an intricate part of the wind waves, on the other hand, the breaking waves have manifested themselves as an elusive object for experimental, particularly field, investigation. Undoubtedly, most of the vast amounts of the time series records of wind waves collected contain breaking waves; nevertheless, most of the conventional study of wave data analysis applied to these time series recordings were performed as if the breaking waves were entirely absent.

Until recently, visual observations of breaking events were the only reliable means of breaking detection (Holthuijsen and Herbers, 1986; Katsaros and Atakturk, 1992; Stolte, 1994; Babanin, 1995; Banner et al., 2000). These very arduous studies involve an observer who counts or marks breaking events by visually monitoring wave probes either in situ or by subsequently viewing videotapes made during observations. Arguably the most accurate, such an approach is nevertheless subject to human error and is too manually intensive and time consuming to be broadly applied in wave research.

Lately, innovative methods using acoustic, optic, or other methods of measuring breaking waves began to be used. Lowen and Melville (1991); Ding and Farmer (1994); Babanin et al. (2001) employed various kinds of acoustic signatures of breaking waves to single them out. Jessup et al. (1997) invented an optical method of quantifying breaking events based on infrared imaging of the skin layer temperature changes associated with the breaking. Gemmrich and Farmer (1999) used void fraction conductivity measurements at sea to describe the scale and occurrence of breaking waves. Smith et al. (1996) and Phillips et al. (2001) studied the speed distribution spectral properties of breaking events using high range resolution radar.

Reliability of direct wave breaking measurements has improved greatly over the years. Most of them remain, however, very expensive. Deployment, maintenance, and exploitation of those sophisticated devices in the open ocean conditions, particularly at extreme wind seas, which are of the most interest, is often a challenging task, clearly impossible to operate on a long-term or even regular basis.

As a recourse to direct measurements, breaking wave studies have generally resorted to theoretical, numerical, and statistical analysis of breaking waves along with supplements of laboratory measurements. Essentially, the endeavors have been directed at studying instability characteristics of wind waves as well as setting wave breaking criteria. LonguetHiggins (1997) has a detailed review of progress made on breaking wave studies over the past three decades. Paradoxically, however, there is still a clear lack of direct field measurements for verification. 
Table 1. Summary of the Black Sea data used; $U_{10}$ is the wind speed at $10 \mathrm{~m}$ height, $k_{p}$ is the peak wave number, $a$ is the standard deviation wave amplitude.

\begin{tabular}{crcc}
\hline Record No. & $U_{10}, \mathrm{~m} / \mathrm{s}$ & $k_{p}, \mathrm{rad} / \mathrm{m}$ & $a, \mathrm{~m}$ \\
\hline 211 & 9.5 & 0.11 & 0.21 \\
238 & 10.7 & 0.12 & 0.22 \\
242 & 10.0 & 0.29 & 0.25 \\
244 & 8.7 & 0.25 & 0.22 \\
\hline
\end{tabular}

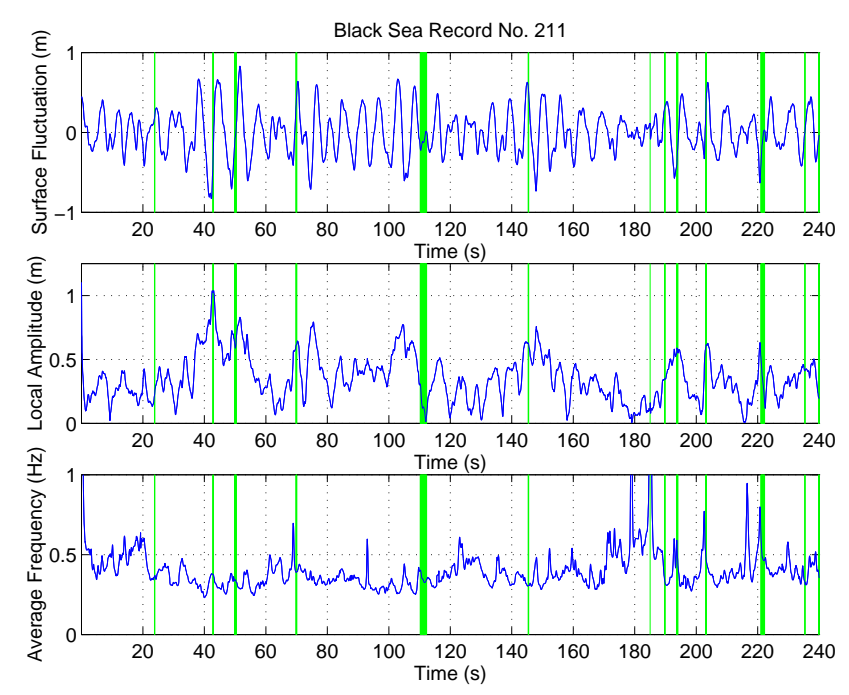

Fig. 1. Black Sea sample results. Vertical bars indicate breaking events.

There remains a need or wishful expectation for a fast and inexpensive method of breaking detection, which would allow the utilization of available wind-wave time series data without relying too much on uncertain geometrical properties of the breaking waves. The advance of wavelet transformation analysis has possibly brought this expectation a little closer to realization.

Mori and Yasuda (1994) considered that there is a sudden surface jump at wave breaking, and interpret it as a shock wave and then defined a shock wavelet spectrum, using discrete wavelet transform and the Meyer (1989) wavelet to detect occurrences of such surface jumps. They verified their method on laboratory-generated waves, breaking randomly, and found a good detection rate. However the use of discrete scales and the ratio of two adjacent wavelet coefficients as a criterion for breaking detection lacks clear physical meaning for the process.

Liu (1993) used continuous wavelet transform and the complex valued Morlet wavelet (Farge, 1992; Liu, 2000) to obtain a time-frequency wavelet spectrum that effectively provided a localized frequency energy spectrum for each data point in a given time series. This spectrum can then be used to define an average wave frequency, $\omega$, and thus combine with the local wave amplitude, $a$, to obtain a local surface acceleration, $a \omega^{2}$, which would be compared to a given lim- iting fraction of gravitational acceleration, $g$, to define the breaking event.

The present paper is aimed at testing the Liu (1993) breaking detection approach on the basis of field data. The data were obtained under a variety of wind-wave conditions in a finite depth environment in Lake George, Australia and in the deep water at the Black Sea.

\section{Experimental data and initial identification of break- ers}

The two data sets included synchronized time series of surface elevations and wave breaking marks. Both have been extensively used to study the breaking statistics and detailed description of the breaking detection procedure, as well as relevant environmental conditions given in Babanin (1995); Babanin and Soloviev (1998); Banner et al. (2000) and Babanin et al. (2001). Here we shall briefly summarize the relevant features.

\subsection{The Black Sea data set}

The Black Sea is a large water basin, extending some $1200 \mathrm{~km}$ east-west and more than $400 \mathrm{~km}$ north-south. Most of the sea is over $1000 \mathrm{~m}$ deep, and therefore wind waves develop in ocean-like conditions. The major difference, compared to the ocean, is the rareness of swell in the Black Sea because of its enclosed location, and an absence of strong surface currents. This makes the Black Sea a convenient site for field observations of deep water waves in their relatively pure state, not perturbed by wave-swell and wave-current interactions.

Four wind wave records, analyzed in the present study, were taken from an oil rig situated on a 30-m deep sea shelf $60 \mathrm{~km}$ offshore in the northwest region of the Black Sea. Fetch and depth environment constituted ideal deep water development conditions, with peak frequencies, $f_{p}$, ranging from 0.16 to $0.27 \mathrm{~Hz}$ (wavelengths $\lambda_{p}$ from 20 to $60 \mathrm{~m}$ ), significant wave heights, $H_{s}$, about $1 \mathrm{~m}$, wind speeds, $U_{10}$, from 8.7 to $10.7 \mathrm{~m} / \mathrm{s}$ at $10 \mathrm{~m}$ height, and mature wave development stages of $U_{10} / c_{p}=1-1.7$, where $c_{p}$ is phase speed of waves, with $f_{p}$ the frequency (Banner et al., 2000). A brief summary of relevant wind-wave properties is summarized in Table 1.

The waves were recorded by an array of high-precision wire wave gauges, deployed beyond the zone perturbed by the platform legs. The breaking events were labelled electronically by an observer (AVB). The observer, located 16 $\mathrm{m}$ above the wave probe array, monitored one of the wave probes and triggered a signal whenever a whitecap of any size occurred at the probe. The signal was recorded synchronously with the wave data. An example of wave record with breaking waves, marked as vertical bars, is shown in the upper panel of Fig. 1. The waves propagate at a speed of $8 \mathrm{~m} / \mathrm{s}$, and thus it is difficult for the observer to place the marks precisely. So the marks, even though they are short, 

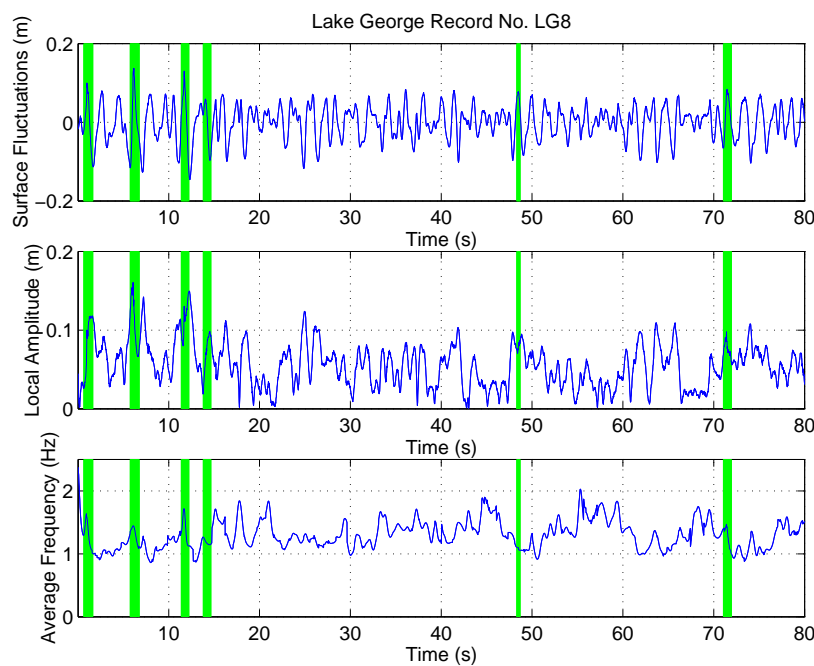

Fig. 2. Lake George sample results. Vertical bars indicate breaking events.

only indicate the presence of breaking waves and not an exact position of whitecapping over the wave phase.

Another set of wave breaking was recorded from a research vessel in the Black Sea and the Mediterranean Sea by means of an accelerometer buoy. Wave measurements by this buoy are described in Babanin et al. (1993), and environmental conditions during the measurements given in Banner et al. (2000). Again, the observer watched the buoy from the vessel and triggered the signal to register the passage of a whitecap over the buoy. Both the ship and the buoy were drifting, the buoy being deployed far beyond the zone perturbed by the ship and connected to the ship by a long, loose cable. The observer was about $10 \mathrm{~m}$ above sea level, allowing a clear view of whitecaps with scales down to the size of the buoy (less than a meter). In the present paper, this set of data was used to obtain the histograms of maximal acceleration in the breaking wave (Fig. 3).

\subsection{The Lake George data set}

The recent Lake George experiment, conducted in a shallow lake in New South Wales, Australia during 1997-2000, was a complex field study designed to simultaneously measure spectral and directional functions of all major source terms that are driving the evolution of wind waves in a finite depth environment (Young et al., 2004). One of the essential goals of the experiment was to adequately detect breaking events, as the breaking determines the whitecapping wave dissipation, enhances the wind input (Banner, 1990; Young and Babanin, 2001) and may effect the wave-bottom interaction at shallow water by means of injecting the turbulent jet into the shear bottom boundary layer.

To reliably measure the wave breaking and associated effects, a number of independent but integrated techniques were employed, as described in Sect. 3.3.1 in Young et al. (2004). Measurements, allowing us to automatically de-

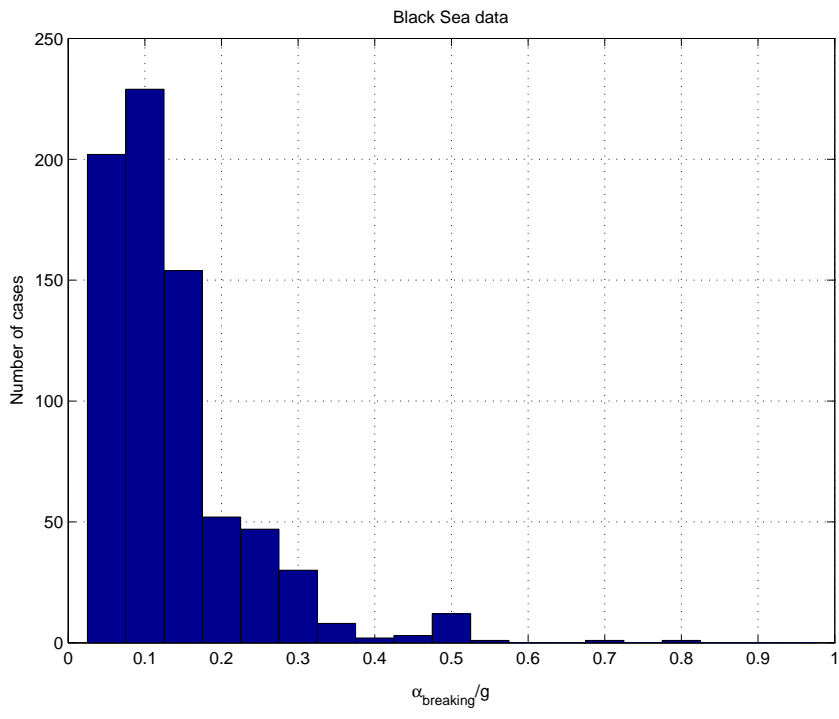

Fig. 3. Histogram of acceleration to gravitation ratio in Black Sea data.

tect the breakers and evaluate their properties, were supplemented by synchronous video recordings of the surface spot of interest.

The data we used here are wave recordings taken by wave resistance probes, and breaking detections were performed by means of bottom pressure probes (Donelan, et al. 2004). The breaking waves generate acoustic pressure and enhanced pressure at high frequencies, which was sensed clearly by the collocated hydrophone (Babanin et al., 2001). The same boosts of pressure were also detected by the pressure probes. Successful detection of the breaking events by the probes was verified with the synchronized video records. The pressure signal was smoothed by applying a running average filter.

An example of such a wave record with the bottom pressure indicated breaking events marked as vertical bars, as shown in the upper panel of Fig. 2. Data records, used in the present study, were conducted during AUSWEX (AUstralian Shallow Water EXperiment), the last stage of the Lake George study when the lake become very shallow (see Table 2). Therefore, these records contrast to those of the deep Black Sea and represent the other end of wave development - bottom-limited and strongly wind-forced waves, under winds of $U_{10} / c_{p}=4.7-7.5$, with peak frequencies $f_{p}=0.53-1.32 \mathrm{~Hz}$ (wave lengths $\lambda_{p}=0.9-3 \mathrm{~m}$ ), and significant wave height $H_{s}$ about $0.1 \mathrm{~m}$.

\section{Principles of wavelet breaking detection technique}

Wavelet analysis has come into flourishing existence over the past two decades and emerged as one of the approaches to extend the popular, century old Fourier Analysis. One of its distinguishing features is its capability to analyze time varying signals with respect to both time and scale, which provides a link to capturing rapid changes in dynamic properties of the 
Table 2. Summary of AUSWEX data. $U_{10}$ is the wind speed at $10 \mathrm{~m}$ height, $k_{p}$ is the peak wave number, $a$ is the standard deviation wave amplitude, $d$ is the water depth.

\begin{tabular}{crccc}
\hline Record No. & $U_{10}, \mathrm{~m} / \mathrm{s}$ & $k_{p}, \mathrm{rad} / \mathrm{m}$ & $a, \mathrm{~m}$ & $d, \mathrm{~m}$ \\
\hline 4 & 6.6 & 7.2 & 0.013 & 0.31 \\
8 & 11.9 & 2.3 & 0.039 & 0.32 \\
9 & 12.0 & 2.1 & 0.034 & 0.29 \\
10 & 8.1 & 3.1 & 0.019 & 0.33 \\
11 & 10.6 & 2.2 & 0.020 & 0.32 \\
14 & 7.1 & 5.6 & 0.015 & 0.27 \\
15 & 7.3 & 2.5 & 0.016 & 0.28 \\
\hline
\end{tabular}

wave surface and associating them with the breaking processes. This Fourier analysis extension is particularly effective using continuous wavelet transform with the complexvalued Morlet wavelet that practically provides a local energy spectrum for every data point of the time series. One of the earlier, successful applications of this kind towards studies of wave spectral properties was done by Donelan et al., 1996), where a method of nonstationary analysis of directional spectra was developed and shown to be able to obtain instantaneous wave propagation directions, amplitude and phase of a spectral frequency component, as well as wave number related time-dependent information.

The classical concept of studying the wave breaking process forged the use of a limiting value of wave steepness beyond which the continuous surface cannot sustain (LonguetHiggins, 1969). Equivalently, assuming a linear dispersion relationship, the wave surface will break when its downward acceleration exceeds a limiting fraction, $\gamma$, of the gravitational acceleration, $g$, i.e. when

$a \omega^{2}>\gamma g$.

When it is applied to a monochromatic wave, this approach is straightforward, even though there are uncertainties about the value of the limiting fraction $\gamma$. In theoretical studies of the limiting steepness Stokes wave, it has generally been assumed that $\gamma=0.5$ (Longuett-Higgins et al., 1963, Snyder and Kennedy, 1983). More recent laboratory studies (Hwang et al., 1989) have shown that $\gamma$ is closer to 0.4. Some field measurements (Holthuijsen and Herbers, 1986) further indicate that the value of $\gamma$ should be even lower.

The greatest difficulty to applying the limiting downward acceleration approach to the real sea waves, however, is not the uncertain value of $\gamma$, but rather the fact that the natural wave fields are multi-scaled and therefore the surface elevation at any point and any instance of time is a superposition of an unlimited number of wave components, and the quantity on the left-hand side of Eq. (1) cannot be readily calculated from a time series of wave data. If it was possible to estimate this quantity, then this simple, familiar notion could be readily used to identify breaking waves in the time series. It is not immediately clear, however, how to pertinently re- solve the local amplitude, $a$, and local frequency, $\omega$, from the measured time series, $X(t)$, of real multi-scaled seas.

Liu (1993) suggested to use the time-frequency wavelet spectrum, $W_{X}(\omega, t)$, to obtain the instantaneous values of effective wave amplitude, $a$, and frequency, $\omega$, from the time series of surface elevations $X(t)$ as

$W_{X}(\omega, t)=\left.\left.\frac{1}{C_{\psi}}\left|\int_{-\infty}^{\infty} X(\tau)\right| \omega\right|^{1 / 2} \psi^{*}[\omega(\tau-t)] d \tau\right|^{2}$,

where $C_{\psi}<\infty$ is the admissibility condition and the function $\psi$ is the mother wavelet. Here we use the Morlet wavelet given by

$\psi(t)=\frac{1}{\pi^{1 / 4}}\left(e^{-i m t}-e^{-m^{2} / 2}\right) e^{-t^{2} / 2}$,

where $m=\pi \sqrt{2 / \ln 2}$ is chosen to fit the wavelet shape.

Once a localized frequency spectrum at each time moment $t_{i}$ is known:

$\Phi_{i}(\omega)=\left[W_{X}(\omega, t)\right]_{t=t_{i}}$

with characteristic wave amplitude and frequency at the measurement point, it replaces the localized spectrum by an equivalent characteristic monochromatic wave.

As the characteristic frequency, average frequency $\sigma$ (Rice, 1954) was chosen:

$\sigma_{i}=\left[\frac{\int_{\lambda \omega_{p}}^{\omega_{n}} \omega^{2} \Phi_{i}(\omega) d \omega}{\int_{\lambda \omega_{p}}^{\omega_{n}} \Phi_{i}(\omega) d \omega}\right]^{1 / 2}$,

where $\omega_{p}$ is the localized frequency at the local energy peak, $\omega_{n}$ is the cutoff frequency, and $\lambda$ is a number which Liu (1993) introduced to denote the start of the frequency range covering the wave breaking process. We generally carried the cutoff frequency up to 2.5 times the peak frequency. The value of $\lambda$ generally lies between 0 and 2. $\lambda=1$, for example, means that we expect waves of peak frequency and higher to be breaking and therefore disregard the contribution of those below $\omega_{p}$ in the determination of the characteristic wave.

For the local amplitude, $a$, Liu (1993) rather crudely used $a_{i}=X\left(t_{i}\right)-\bar{X}$ for an initial application. A more tenable approach will be applied in this paper which is based on considering the case of a simple monochromatic wave that has an acceleration $A \sigma^{2} \cos (\omega t+\varphi)$ to infer that an appropriate characteristic amplitude at local time $t_{i}$ should be given as

$a_{i}=A_{i} \cos \left(p_{i}\right)$,

where the local amplitude $A_{i}$ is given from the analytic envelope signal of $X\left(t_{i}\right)$, obtained by means of Hilbert transform:

$A_{i}=\left|\operatorname{Hilbert}\left(X_{i}\right)\right|$

and local phase, $p_{i}$, can be obtained from the wavelet spectrum, $W_{X}(\omega, t)$.

In order to obtain the phase information of the time series, the mother wavelet to be used should necessarily be a complex one, such as the Morlet wavelet shown above, which is 
what we used here. So the calculation of the phase is given as

$p(\omega, t)=\tan ^{-1}\left\{\frac{\mathfrak{\Im}\left[W_{X}(\omega, t)\right]}{\Re\left[W_{X}(\omega, t)\right]}\right\}$

with the wavelet phase spectrum at each $t=t_{i}$ over the same range between $\lambda \omega_{p}$ and $\omega_{n}$.

Sample results of average frequency and local amplitude based on wavelet spectrum analysis, as obtained from Eqs. (5) and (6) respectively, using $\lambda=1$, are shown in the middle and bottom panels of Figs. 1 and 2, respectively. There remains to be determined the limiting fraction, $\gamma$, as the threshold for wave breaking that can be rendered through assimilation with measured data.

\section{Comparison of the experimental and wavelet detec- tion breaking statistics}

Determination of the limiting fraction $\gamma$ in deep water is based both on direct measurements of surface acceleration in the breaking waves and on comparisons of the measurements and the wavelet breaking detection techniques to provide the same breaking statistics. Feasibility of the approach is verified by means of predicting shallow water $\gamma$ on the basis of knowledge of the deep-water limiting fraction. A reality check is also discussed.

\subsection{Experimental perspectives}

Even though recent advances in the wave breaking studies indicate that no wave surface properties may serve as a criterion for the wave's ability to break (see, for example, Banner and Tian, 1998), it is clear that once the wave is breaking, the downward acceleration of the water particles on the breaking crest will be determined by a ratio $\gamma$ in connection with the centrifugal acceleration of the particle and gravitational acceleration $g$. The exact measure of $\gamma$, however, is rather elusive and yet to be ascertained. As it was mentioned above, in classical studies (Longuet-Higgins et al., 1963; Snyder and Kennedy, 1983), it has been enacted generally as $\gamma=0.5$, although indirect inference of the acceleration based on laboratory (Hwang et al., 1989) and field measurements (Holthuijsen and Herbers, 1986) indicate that the value of $\gamma$ should be 0.4 or even lower.

At the Black Sea measurement, some of the breaking waves were measured by an accelerometer, and therefore direct estimates of maximal acceleration $\alpha_{\text {breaking in such a }}$ wave are available. A histogram of the distribution of these estimates for 742 breaking events is shown in Fig. 3.

To define the limiting fraction $\gamma$, we are interested in the maximal detected value of accelerations $\alpha_{\text {breaking. While be- }}$ ing on a breaking wave, the accelerometer was not necessarily located at a point of maximal acceleration, and therefore not all the events depicted in Fig. 3 are indicative of the limiting acceleration sought after to define $\gamma$.

As can be seen from the histogram in Fig. 3, $\alpha_{\text {breaking val- }}$ ues of up to $0.8 \mathrm{~g}$ were detected, although the number of
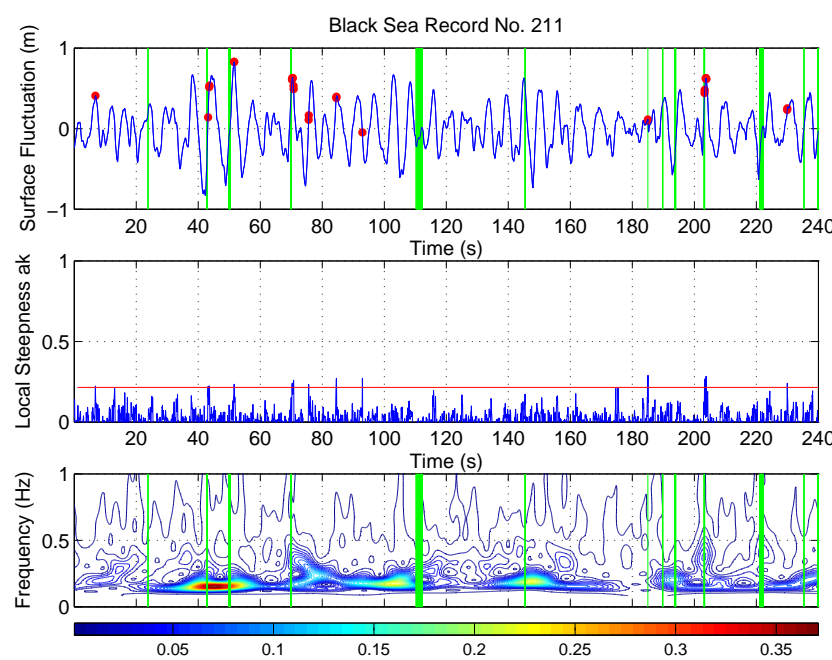

Fig. 4. (a) Comparing breaking events detected from wavelet approach in red dots with Black Sea measurement in green vertical bars. (b) Local steepness $a k$. The straight line shows $\gamma=0.2$, the chosen threshold value. (c) Instantaneous wave spectra in relative units (colour scale below). The horizontal axis is the time axis, same as in the top two panels.

those events is very low and their statistics are poor. Occasional high values of the acceleration could have been registered if the accelerometer was shaken by, for example, a direct hit by a plunging breaker jet or by a jerk of the cable connecting the buoy to the mother ship. The histogram shows that continuous distribution with reliable statistics of

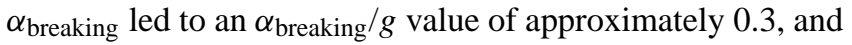
we shall use this as a reference value for $\gamma$ in deep water. The limiting fraction $\gamma$ in shallow water is expected to be different due to reasons to be discussed below in Sect. 4.3.

\subsection{A practical approach}

Following the supposition inferred in Sect. 3, our essential task basically boils down to finding the pertinent values for $\lambda$ and $\gamma$ used to detect wave breaking events based on a wavelet approach. Amidst the redundant choices and combinations of the values of $\lambda$ and $\gamma$, we settled on a plausible and practical approach: to match, as closely as possible, the number of breaking waves estimated from the wavelet approach for a time series with the number of the measured breaking waves. In so doing, for each available time series data set, we can readily resolve a rational $\gamma$ value from a given $\lambda$ value. A physically sound value of $\lambda$, in turn, can be chosen on the basis of knowledge of the lower bound of the frequency scale of waves which are expected to break. This knowledge is available for both deep water breakers (for example, Banner et al., 2000) and finite depth breakers (Babanin et al., 2001).

The top panels of Figs. 4 and 5 present the results of the applications of this approach to the sample cases in the Black Sea and Lake George, shown in Figs. 1 and 2, respectively. The results were obtained for $\lambda=1$. With measured breaking 

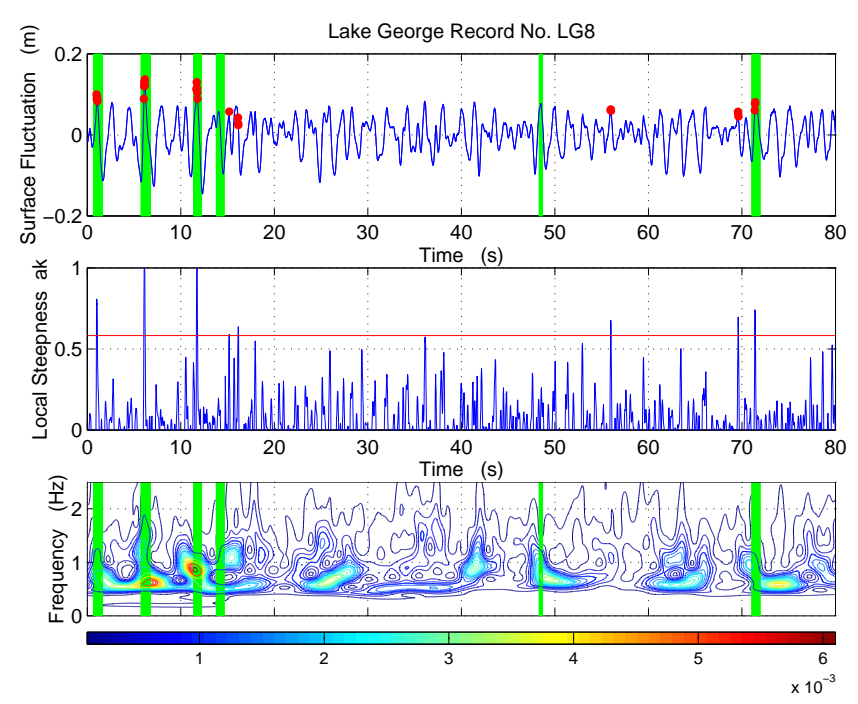

Fig. 5. (a) Comparing breaking events detected from wavelet approach in red dots with Lake George measurement in green vertical bars. (b) Local steepness $a k$. The straight line shows $\gamma=0.6$, which is the chosen threshold value. (c) Instantaneous wave spectra in relative units (colour scale below). The horizontal axis is the time axis, same as in the top two panels.

waves shown as green vertical bars, breaking events detected by the wavelet approach are shown by the red dots. For reference, the figures also include plottings of corresponding local steepness, $a k$, obtained through Eqs. (1), (5) and (6) and given in the middle panel, with the resolved $\gamma$ value plotted as the red line, and the corresponding contours of wavelet spectrum displayed in the lower panel for reference.

An examination of the results indicates that the wavelet approach along with the measurements are generally successful in capturing breaking wave events at many occasions, although at some other occasions one of them fails to detect a breaker while the other indicates that the breaking has occurred. Since the measurement is also basically an approach to capture breaking events, it is not unexpected that both the measurement and the wavelet approach should be anticipated as relevant to different phases of wave breaking.

There is, at the present, no widely established classification of wave-breaking phases, but we may envision a single breaking event as passing through several distinctive stages from both the external appearances of the breaking and the underlying physics involved. These stages are incipient breaking, developing breaking, subsiding breaking, and residual breaking.

At the incipient stage, the wave starts to find that its continuous surface becomes strenuous to sustain, so it is about to break or has just started breaking. An incipient breaker does not have much whitecapping coverage as the breaking crest does not arise to rapidly overturn along its entire length. This is how Phillips et al. (2001) described the development of the crest breaking: "A single breaking event is generally initiated at some point on the wave crest and spreads laterally so that its average length is of the order of half its ultimate length, the width of the broken patch". At this stage, the wavelet method will detect the breaking, because the downward acceleration will clearly exceed the threshold value, while the visual technique implemented at the Black Sea and the acoustic technique implemented at Lake George will not. The two experimental techniques make use of the whitecapping occurrence at the basic measurement spot and, unless the incipient point of the breaking will happen exactly over the spot, they will fail to detect the wave crest as a breaking crest.

The developing stage is characterized by the lateral spread of a breaking with a higher probability of whitecapping appearance for the crest to pass over the measurement spot, so a developing breaker should be readily detected by the whitecap-oriented measurements. But the developing breaker also exhibits an increasing of wave front steepness until it subsides. Rapp and Melville (1990, Subsect. 3.4) defined the front steepness as the ratio of crest-to-frontzerocrossing height to crest-to-front-zerocrossing length and showed that it is larger, compared to the incipient breaking front steepness, for both spilling and plunging breakers. Even though the front steepness is not unambiguously linked to the maximal instantaneous downward acceleration along the profile of a characteristic wave Eqs. (5)-(8), this is an indication that the overreaching acceleration values Eq. (1) may persist through the developing stage, and thus the developing breaker will be detected by the wavelet method as well.

The relaxing or subsiding stage of breaking has not received as much attention in the literature as the developing breaking. Therefore, it is not quite clear, for example, what will happen to the breaking crest once it has reached its maximal length, according to Phillips et al. (2001), or when the front steepness of the breakers, described by Rapp and Melville (1990), starts to decrease. But at some stage it will start to decrease. For the Black Sea waves, shown in the top panel of Fig. 4, mean front steepness was 0.045. The second and the third breakers, picked up by the visual method, have a front steepness of 0.052 and 0.075 , which is greater than the mean steepness as one can intuitively expect for a breaking wave. The first breaker, however, which was also detected visually because a whitecapping crest propagated past the measuring wave probe, has a front steepness of 0.011 , well below the mean wave steepness. Clearly, this broken wave, which still carries along a whitecapping patch, is not expected to be detected by the wavelet method based on Eq. (1) criterion.

During the final stage of breaking, whitecaps are left behind and are decreasing in size as entrained bubbles rise to the surface but spatial evolution of mixing continues as the turbulent front is moving downstream (Rapp and Melville, 1990; Melville and Matusov, 2003). This stage will not be detectable by either wavelet or whitecapping oriented measurement approaches and therefore has no significance for the current study.

In summary, we would like to point out that we expect both the breaking measurements and the wavelet approach to 
detect the same breaking events only at the developing stage of the breaking phases. The incipient breakers will be detected by the wavelet method and will not be detected by the measurements. The subsiding breakers, on the other hand, will be detected by the measurements, whereas the wavelet method will fail to pick them up.

The relative duration of the different breaking phases is not clear, especially those of the incipient and subsiding phases. If we assume that the durations are similar, then the breaking statistics, i.e. number of breakers detected on the basis of measurements and those obtained by the wavelet approach, will be the same even though there will be no $100 \%$ match of waves indicated as breakers. There is no experimental evidence available to support or disprove this assumption. When more evidence becomes available, this assumption may be corrected or modified. For the time being, however, we shall presume the relative duration of the incipient and subsiding phases to be equal and make use of this assumption for calibration of the wavelet method based on the experimental breaking statistics.

As one might have expected, there are breaking cases that our wavelet approach captured, there are breaking cases the wavelet approach did not capture, and there are cases that the approach anticipated as breaking but were not realized by the measurement. They are all attributes of the wave breaking phases. Therefore, it must be unrealistic to expect perfect matchings between the measurement and the detection from the wavelet approach. Nevertheless, the approach of matching breaking percentages as we used here would be the only rational course to pursue toward practical applications. Results of $\gamma$ value assessments for different values of $\lambda$ based on applications of the matching breaking percentage approach to the Black Sea and Lake George data are given in Tables 3 and 4 , respectively.

Another interesting insight provided by the wavelet method pertains to the relation between the wave breaking and wave groupiness. Donelan et al. (1972), followed by a number of experimental and theoretical research (Holthuijsen and Herbers, 1986; Banner and Tian, 1998; among others), reported that the majority of the breaking events take place within the group structure close to the peak of the group envelope. Figures 1-4 show that breaking does happen beyond the obvious groups of dominant waves, and the instantaneous spectra plotted in Figs. 3-4 indicate why this may be the case.

Once the limiting acceleration criterion Eq. (1) is applied to the instantaneous wave Eqs. (5)-(8), which is characteristic of the instantaneous wave spectrum shown in the bottom panels of Figs. 3-4, occurrence of the breaking event will be dependent on the product of characteristic amplitude $a$ and characteristic average frequency $\sigma$ squared. The peak of the envelope of dominant waves will give rise to the amplitude $a$, but not to the frequency $\sigma$. If the amplitude rise results in overshooting the threshold value Eq. (1), the wave will break at the wave group crest. If, however, deformation of the instantaneous spectrum leads to the rise of the average frequency $\sigma$, this will indicate wave breaking far from the en-

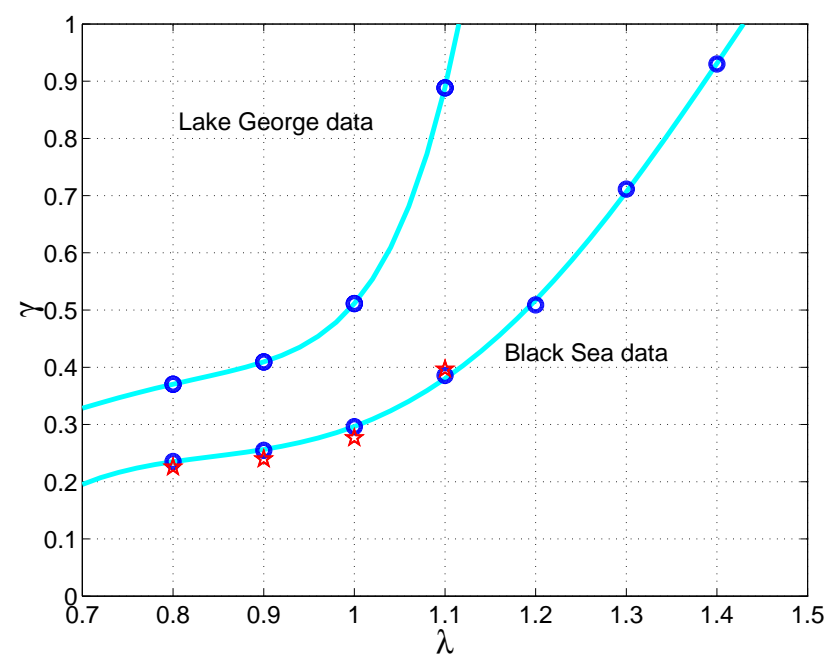

Fig. 6. The lambda-gamma graph. Circles indicate data from Tables 3 and 4. Stars show conversion of finite depth values of $\gamma$ according to Eqs. (9)-(12).

velope crest and in fact may happen outside of a visible wave group. Such events can be seen around the 70th and the 205th seconds of the Black Sea record in Fig. 4, where the second higher-frequency peak appears in the instantaneous spectrum and shifts up the average frequency; respective breakers are detected by both measurements and the wavelet method.

The secondary peak is a rather permanent feature of shallow water spectra, and the Lake George spectra in particular. Therefore, one would expect wave breaking to occur more frequently beyond obvious group structure at finite depths, and to occur more frequently in general. The first expectation is supported by Fig. 5, where many breakers are associated with rising instantaneous secondary peaks, and the second expectation relates to the known fact that breaking rates are much higher in the finite depths, compared to the deep water (for example, Babanin et al., 2001). Again, we do not claim to have found a cause for wave breaking, but only an indicator, as the reason for respective pulsation of the instantaneous wave spectrum is determined by the underlying physics, like that of the evolution of nonlinear wave groups of Banner and Tian (1998), and further discussion is beyond the scope of the present paper.

\subsection{The $\gamma-\lambda$ graph}

We plotted the mean values of $\gamma$ shown in Tables 3 and 4 versus the corresponding values of $\lambda$ to yield two distinctive and surprisingly smooth $\gamma-\lambda$ curves for shallow (Lake George) and deep (Black Sea) water conditions. The curves are shown in Fig. 6.

While the clear distinction between the shallow and deep water conditions is by no means unexpected, it presents a tangible challenge for an analytical and physically sound interpretation of these results. Previously, we postulated that the limiting downward acceleration is determined by the balance 
Table 3. Adapted $\gamma$ values from Black Sea data.

\begin{tabular}{llllllll}
\hline Rec. No. & $\lambda=0.8$ & $\lambda=0.9$ & $\lambda=1.0$ & $\lambda=1.1$ & $\lambda=1.2$ & $\lambda=1.3$ & $\lambda=1.4$ \\
\hline 211 & 0.1800 & 0.1940 & 0.2150 & 0.2630 & 0.2950 & 0.4100 & 0.6200 \\
238 & 0.2050 & 0.2230 & 0.2550 & 0.3300 & 0.4350 & 0.6400 & 0.8000 \\
242 & 0.2878 & 0.3070 & 0.3650 & 0.4750 & 0.6280 & 0.8750 & 1.1000 \\
244 & 0.2690 & 0.2950 & 0.3500 & 0.4750 & 0.6780 & 0.9200 & 1.2000 \\
Mean & 0.2354 & 0.2547 & 0.2963 & 0.3857 & 0.5090 & 0.7113 & 0.9300 \\
Std. Dev & \pm 0.0512 & \pm 0.0549 & \pm 0.0728 & \pm 0.1066 & \pm 0.1770 & \pm 0.2354 & \pm 0.2676 \\
\hline
\end{tabular}

Table 4. Adapted $\gamma$ values from AUSWEX data.

\begin{tabular}{lllll}
\hline Record No. & $\lambda=0.8$ & $\lambda=0.9$ & $\lambda=1.0$ & $\lambda=1.1$ \\
\hline 4 & 0.3480 & 0.3825 & 0.4800 & 0.8170 \\
8 & 0.4540 & 0.4900 & 0.5820 & 1.0500 \\
9 & 0.4600 & 0.5150 & 0.6700 & 1.2355 \\
10 & 0.2900 & 0.3280 & 0.4350 & 0.7680 \\
11 & 0.3180 & 0.3500 & 0.4250 & 0.6690 \\
14 & 0.2855 & 0.3180 & 0.4050 & 0.7070 \\
15 & 0.4370 & 0.4820 & 0.5820 & 0.9730 \\
Mean & 0.3704 & 0.4094 & 0.5113 & 0.8885 \\
Std. Deviation & \pm 0.0779 & \pm 0.0838 & \pm 0.1006 & \pm 0.2059 \\
\hline
\end{tabular}

of the gravitational and centrifugal forces; the difference of the two curves should be possible to explain if we take into account the difference between the centrifugal accelerations for deep water $\left(\alpha_{\text {deep }}\right)$ and finite depth $\left(\alpha_{\text {shallow }}\right)$ waves of the same frequency $\omega$. Conspicuously, this can be obtained if we consider what happens at the wave crest.

We expect that when the wave surface breaks once it can no longer sustain itself for some reason, the downward acceleration at the surface must have exceeded some threshold level that we wish to resolve. Effective downward acceleration of the real physical particles at the crest is the difference between gravitational acceleration and centrifugal acceleration caused by the motion of the particle along its orbit. The latter is given by $\omega^{2} R$, where $R$ is the radius of curvature of the motion of a particle on the breaking crest:

$$
\frac{\alpha_{\text {deep }}}{\alpha_{\text {shallow }}}=\frac{\omega^{2} R_{\text {deep }}}{\omega^{2} R_{\text {shallow }}}=\frac{R_{\text {deep }}}{R_{\text {shallow }}},
$$

where $R_{\text {deep }}$ and $R_{\text {shallow }}$ are now radii of curvature at the highest points of wave orbits, configured as circles in deep water and ellipsoids in finite depth. The curvature of the twodimensional curve defined by the $y=y(x)$ function is

$$
\frac{1}{R}=\frac{\frac{d^{2} y}{d x^{2}}}{\sqrt{\left[1+\left(\frac{d y}{d x}\right)^{2}\right]^{3}}},
$$

(see, for example, Korn and Korn, 1968).

Now in deep water we have

$R_{\text {deep }}=a$, where $a$ is the local wave amplitude. For the shallow water with water depth $d$ it can be shown that

$R_{\text {shallow }}=a\left\{\frac{\cosh ^{2}[k(d+a)]}{\sinh [k(d+a)] \sinh (k d)}\right\}$.

In essence, the orbits in shallow water are horizontally extended so that the centrifugal accelerations are larger for the same values of amplitude $a$ and frequency $\omega$, and thus the effective downward acceleration is smaller, which, in turn, leads to larger values of $\gamma$ for shallow water cases. Clearly, a correction can be obtained from the ratio of $R_{\text {shallow }} / R_{\text {deep }}$. Applying this formulation to the four $\gamma$ values of Lake George data at $\lambda$ values of $0.8,0.9,1.0$, and 1.1 in Fig. 6 successfully adjusted them closer to the deep water cases as shown by the plottings of the four open stars.

The significance of this result extends deeper than merely resolving the differences of the two curves. Our ability to bring together the two distinctly different results, obtained from explicitly different wave environments, by applying the principles of physics, shows that the wavelet breaking detection approach based on the limiting acceleration concept has a clear physical meaning rather than just a technical measure. It allows us to apply a single approach for both deep and shallow waters and thus translate derived parameters of $\gamma$ and $\lambda$ from one environment into another.

It is also of interest to note that based on the Black Sea acceleration measurement, as shown in Fig. 3, we have picked a reference value of 0.3 for $\gamma$; now the deep water $\gamma-\lambda$ curve based on Black Sea breaking wave measurements $\gamma$ is shown 
to be 0.3 at $\lambda=1$. Thus, $\lambda=1$ would be another appropriate reference value to use, particularly as in Fig. 6 this value also corresponds to the transition of the translated finite depth values of $\gamma$ from underestimating to overestimating their deep water counterparts. It is certainly plausible to consider that the wave breaking process covers the high frequency side of the spectrum, including the extent at peak energy, and it agrees with the deep water study of Banner et al. (2000) and with the finite depth study of Babanin et al. (2001), which both concluded that peak waves do break. A more refined analysis may require an introduction of a wave age dependence for $\lambda$ as, according to Banner et al. (2000), the peak waves do not break if the waves are old enough and thus $\lambda>1$ may be expected in such circumstances, but this is left for future investigations.

\subsection{A reality check-final counts of breaking waves}

Now that we have devised a feasible rational approach that works reasonably well and leads to some encouraging novel results, what we are not yet certain of is just exactly how well does it works. To make an objective assessment of the performance of our approach, we simply picked the $\gamma$ values for $\lambda=1$ given in Tables 3 and 4, applied them to our available data sets, and counted the resulted breaking event matchings between the wavelet-approach detection and the measurements. The results of our final counts of breaking cases are shown in Fig. 7.

We have two kinds of counts shown here: the total breaking counts, shown by the open triangles, and counts of perfect matchings, shown by the triangles with a circle inscribed inside. The Lake George shallow water cases are shown by triangles with vertex points up, and the Black Sea deep water cases are shown with triangular vertex points down.

The straight line through the points of total breaking counts in the figure is the one-one correlation line, since the adaption of the $\gamma$ value was based on a closer matching of breaking percentages. The points follow the line with a 0.94 correlation coefficient and a 0.19 deviation index (D.I.) defined as

D.I. $=\frac{1}{M} \sum \frac{\left|N_{\text {wavelet }}-N_{\text {measured }}\right|}{N_{\text {measured }}}$,

where $M$ is the total number of records available and $N_{\text {measured }}$ and $N_{\text {wavelet }}$ are the breaking cases detected by the measurements and by the wavelet method, respectively. The general ability of the wavelet method to predict the same breaking statistics as the measurements, based on single point observations, is very good. This implies that the wavelet technique can be applied to surface elevation time series to estimate the breaking statistics at those wave scales which have a reasonably smoothly-resolved wave profile for a given sampling frequency, i.e. have a large enough number of surface elevation readings per front face of the wave and around the wave crest.

As seen in Fig. 7, the wavelet analysis somewhat overestimates the total breaking percentage measured at Lake George

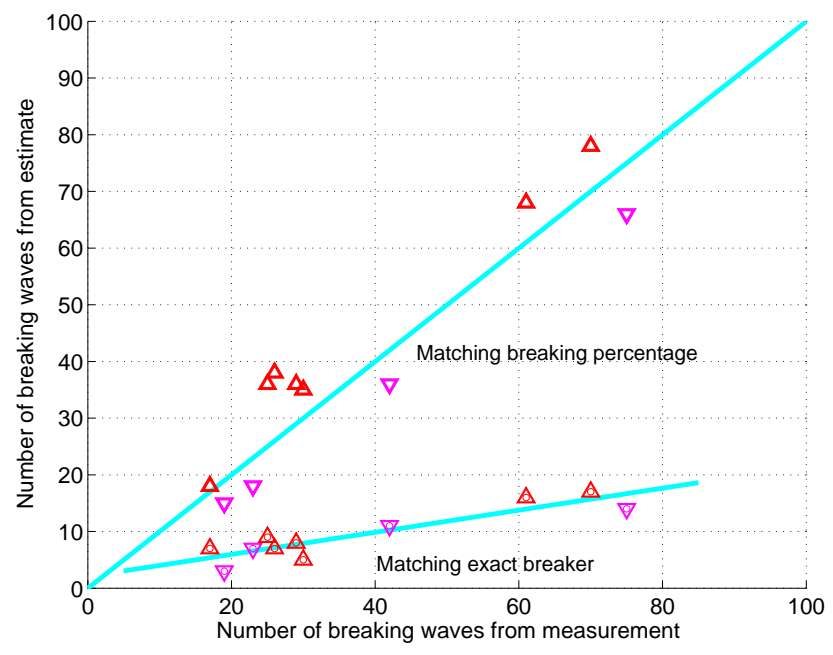

Fig. 7. Final counts of breaking cases.

and slightly underestimates that measured at the Black Sea. At Lake George, the acoustic method used only allowed the detection of the dominant breakers in $\pm 30 \%$ vicinity of the spectral peak (Babanin et al., 2001), and therefore the wavelet method, whose performance is not limited by the spectral peak band, may have an extra number of breakers contributing into the total statistics. At the Black Sea, on the contrary, the breakers were detected visually and did not have an upper-frequency bound, except that of the physical capability of the observer to actually see small whitecaps. Therefore, the wavelet detection method may have failed for small breakers whose profile was not sampled well enough but whose whitecapping was detected by the observer. This would lead to an underestimation of the deep sea breaking rates. Another possible contributor into the observed deep water-shallow water variation in performance can be a difference in relative durations of the breaking phases, as discussed below.

The straight line through the perfect matching counts is a best linear fit line of the data. This line, to a large extent, is the ultimate reality we have to face, i.e. the number of perfect matchings we can really amass. In terms of percentages, the results ranges from a low of 16.7 percent (LG14) to a high of 41.2 percent (LG9), with an average of 28.4 percent of perfect matching cases. At first sight this result is rather discouraging, and we may have harbored an aspiration for more perfect matchings. On the other hand, by taking into consideration the possible breaking stages that we alluded to before, this result may be just as good as what we can expect. While the results might be less than exhilarating, we may have just revealed the unvarnished reality of the general threshold-based approach for breaking wave detection.

The slope of the lower line brings up an interesting conclusion regarding the duration of the breaking phases introduced in Sect. 4.2. This slope is roughly $1 / 3$ of the one-toone match curve, and that means that the second breaking stage, which corresponds to both the methods detecting the 
same breakers, lasts for approximately $1 / 3$ of the breaking duration. Since we have supposed earlier that the first and the third stages are of equal duration, that would mean that all three breaking phases are of approximately the same duration. As we introduced the phases on the basis of physical and external appearance, this result makes the classification of phases also viable from a practical point of view. A breaking wave, having passed through one-third of its breaking activity in progress, may well be considered as having transited into the next breaking phase.

The Black Sea and Lake George points are scattered evenly around the lower line, which means that the second phase duration does not depend on whether the breaking occurs in deep or in shallow water. If, however, the relative duration of the first and third breaking phases changes in finite depth compared to the deep water, one should expect separation of the Black Sea and Lake George data relative to the mean upper curve. If, for example, in deep water, the incipient phase, which corresponds to the wavelet method detecting the breaking and the measurements failing, is shorter than the subsiding stage, which corresponds to the wavelet method failing and the measurements successful in breaking detection, then for the Black Sea data, the wavelet technique should underestimate the total breaking rates compared to the measurements, as is the case in Fig. 7. Similarly, if in shallow water the first stage is longer than the third stage, the Lake George points should demonstrate an overestimation once the wavelet technique is applied, as is again the case in Fig. 7. The fact that there are only small deviations in the experimental points from the upper one-to-one correlation line provides indirect support for the assumption, made in Sect. 4.2, about the relative equality of the duration of the first and third phases.

\section{Concluding remarks}

Wavelet transform has provided, perhaps for the first time, an opportunity to look at each individual wave crest in a time series of wave data and assess whether or not it might be a breaking wave. The approach, however, is by no means free from redundancy. The development of the approach will be futile without the counsel of actual measurements. It is the availability of actual field measurements that puts us on the right track and led our study to fruition. In the process, we learned the strengths and weaknesses of the wavelet approach, which can be served as bearings for further continued studies. Here we summarize the highlights of our study:

- We have developed a wavelet-based approach for detecting breaking waves in a given time series of surface fluctuations. Within the realm of our results, the approach is shown to be able to produce the same breaking statistics as field measurements of wave breaking conditions based on the detection of whitecaps at a fixed point of observation. This new approach requires only access to a wave time series to produce a rational esti- mate of breaking waves that could not have been done previously.

- The approach uses the classical limiting downward acceleration concept, developed primarily for monochromatic waves (e.g. Longuet-Higgins, 1969). With wavelet transform and time-frequency analysis, this concept can now be extended to spectral waves, when an instantaneous wave spectrum is replaced by an instantaneous characteristic wave Eqs. (5) - (8), and applied to actual sea wave measurements. The results can be interpreted through basic wave physics and a limiting value of the acceleration that was obtained from available field measurements.

- The approach is applicable to both deep water and finite depth environments.

- Analysis of the results led us to propose a classification of wave breaking phases. They are incipient breaking, developing breaking, and subsiding breaking. The relative duration of each of the three phases is shown to be approximately equal.

In general, we think the approach can be used as a rightful tool for breaking wave detections in a time series. Hopefully the wavelet approach and the results we present here can be effectively used for implementing further practical approaches for resourceful detection of wave breaking events and the study of wave breaking statistics in the vastly available time series data of ocean waves.

Acknowledgements. GLERL Contribution No. 1328 AVB gratefully acknowledges the financial support of the U.S. Office of Naval Research (grant no. N00014-00-1-0012) and of the Australian Research Council (grant no. A00102965).

Topical Editor N. Pinardi thanks M. Rixen and another referee for their help in evaluating this paper.

\section{References}

Babanin, A. V., Verkeev, P. P., Krivinskii, B. B., and Proschenko, V. G.: Measurement of wind waves by means of a buoy accelerometer wave gauge. Phys. Oceanogr., 4, 387-393, 1993.

Babanin, A. V.: Field and Laboratory Observations of Wind Wave Breaking. In The Second International Conference on the Mediterranian Coastal Environment, Tarragona, Spain, October 24-27, 1995, Ed. E.Ozhan, Autoritat Potuaria de Tarragona, Spain, 3, 1919-1928, 1995.

Babanin, A. V. and Soloviev, Yu. P.: Field Investigation of Transformation of the Wind Wave Frequency Spectrum with Fetch and the Stage of Development. J. Phys. Oceanogr., 28, 563-576. 1998.

Babanin, A. V., Young, I. R., and Banner, M. L.: Breaking Probabilities for Dominant Surface Waves on Water of Finite Constant Depth. J. Geophys. Res., 106, C6, 11 659-11 676, 2001.

Banner, M. L.: The influence of wave breaking on the surface pressure distribution in wind-wave interactions. J. Fluid Mech., 211, 463-495, 1990. 
Banner, M. L. and Tian, X.: On the determination of the onset of wave breaking for modulating surface gravity water waves. J. Fluid Mech., 367, 107-137, 1998.

Banner, M. L., Babanin, A. V., and Young, I. R.: Breaking Probability for Dominant Waves on the Sea Surface. J. Phys. Oceanog., 30, 3145-3160, 2000.

Ding, L. and Farmer, D. M.: Observations of breaking surface wave Statistics,. J. Phys. Oceanogr., 24, 1368-1387, 1994.

Donelan, M. A., Longuett-Higgins, M. S., and Turner, J. S.: Whitecaps. Nature, 239, 449-451, 1972.

Donelan, M. A., Drennan, W. M., and Magnusson, A. K.: Nonstationary analysis of the directional properties of propagating waves, J. Phys. Oceanogr., 26, 1901-1914, 1996.

Donelan, M. A., Babanin, A. V., Young, I. R., Banner, M. L., and McCormic, C.: Wave follower field measurements of the wind input spectral function. Part I. Measurements and calibration, J. Atmosp. Oceanic Technol., in press, 2004.

Farge, M.: Wavelet transforms and their applications to turbulence, Ann. Rev. Fluid Mech., 24, 395-457, 1992.

Gemmrich, J. R. and Farmer, D. M.: Observations of the scale and occurrence of breaking surface waves. J. Phys. Oceanogr., 29, 2595-2606, 1999.

Hwang, P. A., Xu, D., and Wu, J.: Breaking of wind-generated waves: measurements and characteristics. J. Fluid Mech., 202, 177-200, 1989.

Holthuijsen, L. H. and Herbers, T. H. C.: Statistics of breaking waves observed as whitecaps in the open sea. J. Phys. Oceanogr., 16, 290-297, 1986.

Jessup, A. T., Zappa, C. J., Loewen, M. R., and Hesany, V.: Infrared remote sensing of breaking waves. Nature, 385, 52-55, 1997.

Katsaros, K. B. and Atakturk, S. S.: Dependence of wave-breaking statistics on wind stress and wave development. In Breaking waves, M. L. Banner and R. H. J. Grimshaw, Eds., Springer, 119132, 1992.

Korn, G. A. and Korn, T. M.: Mathematical handbook for scientists and engineers. McGraw-Hill Book Company, 832 pp, 1968.

Liu, P. C.: Estimating breaking statistics from wind-wave time series data. Ann. Geophys., 11, 970-972, 1993.

Liu, P. C.: Wavelet transform and new perspective on coastal and ocean engineering data analysis in Advances in Coastal and Ocean Engineering, (P. L. F., Liu, ed.), World Scientific, Volume 6, 57-101, 2000.

Longuet-Higgins, M. S.: The generation of capillary waves by steep gravity waves. J. Fluid Mech., 16, 138-159, 1963.
Longuet-Higgins, M. S.: On wave breaking and the equilibrium spectrum of wind-generated waves. Proc. Rpy. Soc., A310, 151$159,1969$.

Longuet-Higgins, M. S.: Progress Toward Understanding How Waves Break. Twenty-First Symposium on Naval Hydrodynamics, National Academy Press, 5-28, 1997.

Lowen, M. R. and Melville, W. K.: Microwave backscatter and acoustic radiation from breaking waves. J. Fluid Mech., 224 601-623, 1991.

Melville, W. K. and P. Matusov: Distribution of breaking waves at the ocean surface. Nature, 417, 61-63, 2003.

Meyer, Y.: Wavelets and operators. In Analysis at Urbana I, (Berkson et al., eds.), London Math. Soc. Lecture Notes, Cambridge University Press, 137, 256-365, 1989.

Mori, N. and Yasuda, T.: Orthonormal wavelet analysis for deepwater breaking waves. In Proceedings of the 24.International Conference on Coastal Engineering, Koba, Japan, Chapter 31, 412-426, 1994.

Phillips, O. M., Posner, F. L., and Hansen, J. P.: High resolution radar measurements of the speed distribution of breaking events in wind-generated ocean waves: surface impulse and wave energy dissipation rates. J. Phys. Oceanogr., 31, 450-460, 2001.

Rapp, R. J. and Melville, W. K.: Laboratory measurements of deepwater breaking waves. Phil. Trans. R. Soc. Lond., A311, 735800, 1990.

Rice, S. O.: Mathematical analysis of random noise. In Noise and Stochastic Processes, N. Wax, Ed., Dover, New York, 133-294, 1954.

Smith, M. J., Poulter, E. M., and McGregor, J. A.: Doppler radar measurements of wave groups and breaking waves. J. Geophys. Res., C101, 14 269-14 282, 1996.

Snyder, R. L., Kennedy, R. M., and Smith, L.: On the Formation of Whitecaps by a Threshold Mechanism. Part I: Basic Formalism. J. Phys. Oceanogr., 13, 1482-1492, 1983.

Stolte, S.: Short-wave measurements by a fixed tower-based and a drifting buoy system. IEEE J. Oceanic Eng., 19, 10-22, 1994.

Young, I. R. and Babanin, A. V.: Wind Wave Evolution in Finite Depth Water. In Proceedings of the 14th Australasian Fluid Mechanics Conference, Adelaide, Australia, 79-86, 2001.

Young, I. R., Banner, M. L., Donelan, M. A., Babanin, A. V., Melville, W. K., Veron, F., and McCormic, C.: An Integrated Study of the Wind Wave Source Term Balance in Finite Depth Water, J. Atmos. Oceanic Technol., in press, 2004. 